\title{
Les fouilles du site de Kabah (Yucatán) : saison 1991
}

Ramón Carrasco V., Josep Ligorred, Eduardo Pérez de Heredia, Antonio Centeno, Fabienne de Pierrebourg

\section{Citer ce document / Cite this document :}

Carrasco V. Ramón, Ligorred Josep, Pérez de Heredia Eduardo, Centeno Antonio, de Pierrebourg Fabienne. Les fouilles du site de Kabah (Yucatán) : saison 1991. In: Journal de la Société des Américanistes. Tome 78 n¹, 1992. pp. 9-29; doi : https://doi.org/10.3406/jsa.1992.1408

https://www.persee.fr/doc/jsa_0037-9174_1992_num_78_1_1408

Fichier pdf généré le 29/03/2019 


\title{
Resumen
}

Las excavaciones del sitio de Kabah (Yucatan) : temporada 1991. Kabah es un sitio mayor de la cultura puuc, la cual se desarrolló en las Tierras Bajas del norte de Yucatan. En la temporada 1991 del proyecto Kabah, se trabajaron las estructuras principales que conforman el Grupo Este y los edificios conocidos como Codz Pop y Manos Rojas. Los resultados de las exploraciones realizadas contradicen la hipótesis segun la cual floreció la cultura puuc repentina y tardiamente (750-1 000 d. C). Un reconocimiento de superficie que tiene como objetivos establecer los limites del sitio y completar el piano de Pollock (1980) empezó durante esta misma temporada, finalmente se excavó también una plataforma habitacional.

\begin{abstract}
Excavations of the site of Kabah ( Yucatan) : season 1991. Kabah is a major site of the Puuc culture which developed in the Northern Maya lowlands. Excavations were carried out in 1991 by the Kabah Project in the buildings of the Eastern Group and those known as Codz Pop and Manos Rojas. The results of explorations of these structures raise doubs concerning the sudden and late development of Puuc culture (750-1 000 AD). A survey to establish the limits of the site and complete the plan drawn up by Pollock (1980) began the same season during which a dwelling platform was also excavated.
\end{abstract}

\section{Résumé}

Kabah ' est un site majeur de la culture Puuc qui s'est développé dans les Basses Terres mayas septentrionales. Les travaux réalisés en 1991 par le Projet Kabah concernent les édifices du Groupe Est et ceux connus sous les noms de Codz Pop et de Manos Rojas. Les résultats de l'exploration de ces structures remettent en question l'hypothèse d'un développement subit et tardif de la culture Puuc en seulement 250 ans (750-1000 ap. J-C). Une reconnaissance de surface visant à déterminer les limites du site et à compléter le plan réalisé par Pollock (1980) a débuté au cours de la même saison et une plate-forme d'habitation a également été fouillée. 


\title{
LES FOUILLES DU SITE DE KABAH (YUCATÁN) : SAISON 1991
}

\author{
Ramón CARRASCO V., Josep LIGORRED, Eduardo PÉREZ de HEREDIA P., \\ Antonio CENTENO et Fabienne de PIERREBOURG ${ }^{*}$
}

Kabah ${ }^{1}$ est un site majeur de la culture Puuc qui s'est développé dans les Basses Terres mayas septentrionales. Les travaux réalisés en 1991 par le Projet Kabah concernent les édifices du Groupe Est et ceux connus sous les noms de Codz Pop et de Manos Rojas. Les résultats de l'exploration de ces structures remettent en question l'hypothèse d'un développement subit et tardif de la culture Puuc en seulement 250 ans (750-1000 ap. J-C). Une reconnaissance de surface visant à déterminer les limites du site et à compléter le plan réalisé par Pollock (1980) a débuté au cours de la même saison et une plate-forme d'habitation a également été fouillée.

\section{Las excavaciones del sitio de Kabah (Yucatán) : temporada 1991}

Kabah es un sitio mayor de la cultura puuc, la cual se desarrolló en las Tierras Bajas del norte de Yucatán. En la temporada 1991 del proyecto Kabah, se trabajaron las estructuras principales que conforman el Grupo Este y los edificios conocidos como Codz Pop y Manos Rojas. Los resultados de las exploraciones realizadas contradicen la hipótesis según la cual floreció la cultura puuc repentina y tardíamente $(750-1000 \mathrm{~d}$. C.). Un reconocimiento de superficie que tiene como objetivos establecer los límites del sitio y completar el plano de Pollock (1980) empezó durante esta misma temporada, finalmente se excavó también una plataforma habitacional.

\section{Excavations of the site of Kabah (Yucatan) : season 1991}

Kabah is a major site of the Puuc culture which developed in the Northern Maya lowlands. Excavations were carried out in 1991 by the Kabah Project in the buildings of the Eastern Group and those known as Codz Pop and Manos Rojas. The results of explorations of these structures raise doubs concerning the sudden and late development of Puuc culture (750-1000 AD). A survey to establish the

* Voir note 1

J.S.A. LXXVIII-I, 1992 : p. 9 a 29. 


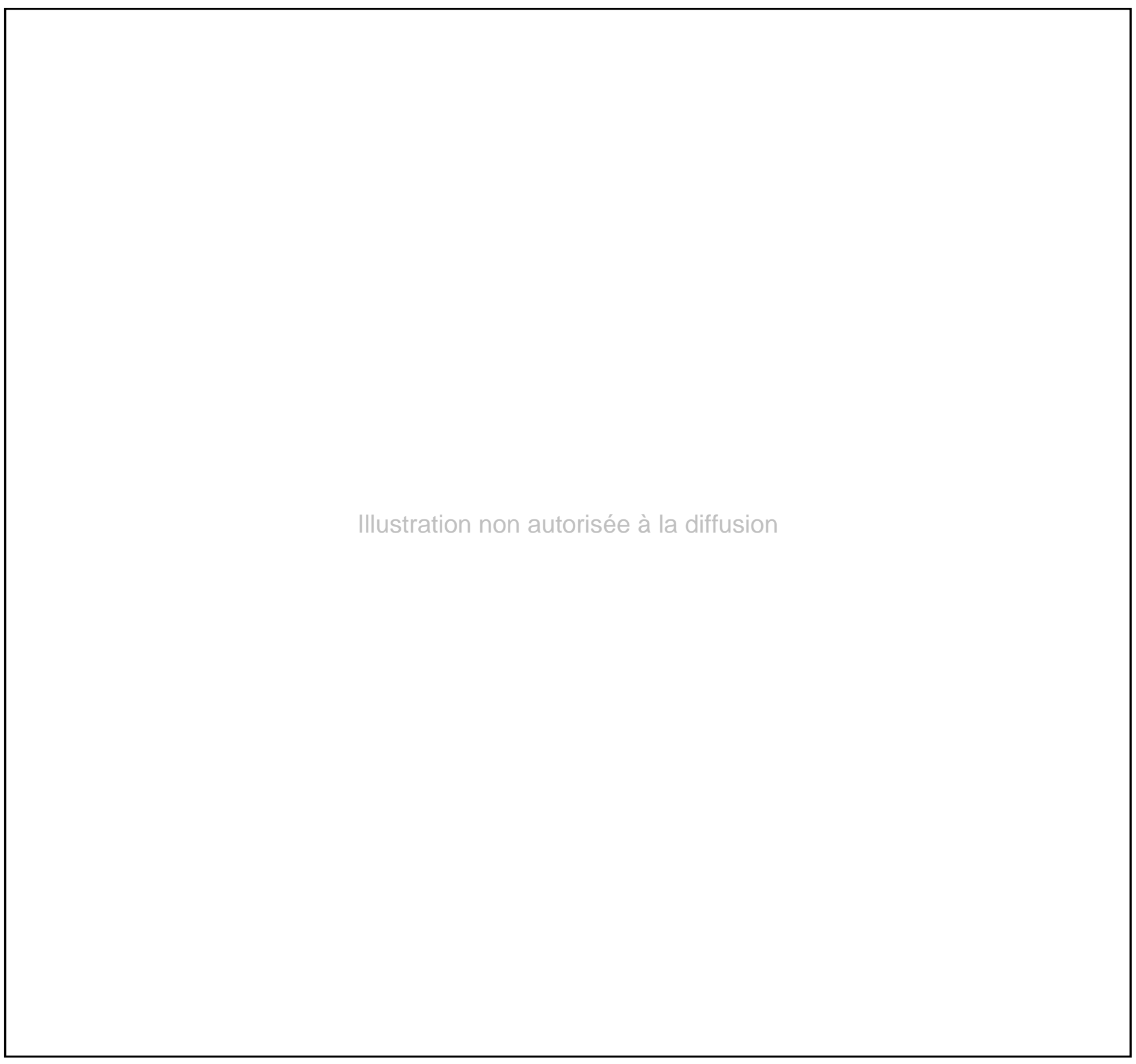

Fig. 1. -- Localisation de Kabah (d'après N. Hammond, 1982) 
limits of the site and complete the plan drawn up by Pollock (1980) began the same season during which a dwelling platform was also excavated.

Localisé entre Uxmal et Sayil, Kabah fait partie du groupe des sites les plus importants de la culture Puuc (fig. 1 et 2$)^{2}$. Cette dernière est surtout connue pour l'excellence de ses réalisations architecturales. Son apogée est situé entre 750 et 1000 de notre ère, intervalle qui correspond à l'horizon céramique Cehpech. La culture Puuc s'est épanouie au nord-ouest de la péninsule du Yucatán, dans une région au relief de type karstique, où alternent des collines calcaires et des dépressions fertiles d'extension variable; dans ce secteur, le réseau hydrographique est totalement souterrain. Les grands sites ont, depuis longtemps, attiré explorateurs et archéologues. Mais de nombreux bâtiments sont encore sous les décombres et de nombreuses questions sur l'histoire, l'organisation sociale, politique et économique de ces sites demeurent sans réponses.

Le Projet Kabah, lancé fin 90 par le Centre Régional du Yucatán (INAH) et dirigé par Ramón Carrasco vise naturellement à une meilleure compréhension du site et de la culture à laquelle il se rattache; nous en présentons ici les premiers résultats. La restauration et la mise en valeur du Groupe Est ont été les priorités du Projet Kabah durant cette saison. L'architecture de ce groupe est un document exceptionnel pour comprendre l'évolution du site et l'histoire de l'aire Puuc. En effet, ses différents monuments reflètent, au cœur d'une même unité, l'évolution architecturale de la région durant le classique final et révèlent, en même temps, des origines plus anciennes. Les édifices baptisés respectivement Codz Pop et Manos Rojas sont des éléments essentiels du site. Leur exploration et leur restauration apportent de nouvelles informations d'ordre politique, rituel et historique. Ces travaux, appuyés par de nouvelles études céramiques, élargissent le cadre chronologique Puuc : ils montrent que la culture puuc n'a pas eu une durée limitée à 250 ans, comme le suggèrent certains travaux, mais que ses origines sont plus anciennes et que des prolongements/réoccupations au Postclassique ne sont pas à exclure. En 1991, a débuté également une reconnaissance de surface pour réviser et compléter le plan établi par Pollock (1980) ${ }^{3}$. Il s'agissait, en particulier, de définir les limites du site pour organiser la protection de la zone archéologique et dévier la route fédérale qui traverse le site en son centre. Etablir un plan détaillé de Kabah devrait aussi et surtout aider à comprendre son organisation interne ; c'est à partir de cela qu'on peut espérer entreprendre la reconstitution des relations démographiques, sociales, politiques et économiques qui unissaient ses habitants. La fouille intensive d'une unité d'habitation périphérique a complété la prospection et abordé un aspect de l'archéologie trop souvent négligé dans cette région: chercher à comprendre la vie domestique des anciens Mayas. 

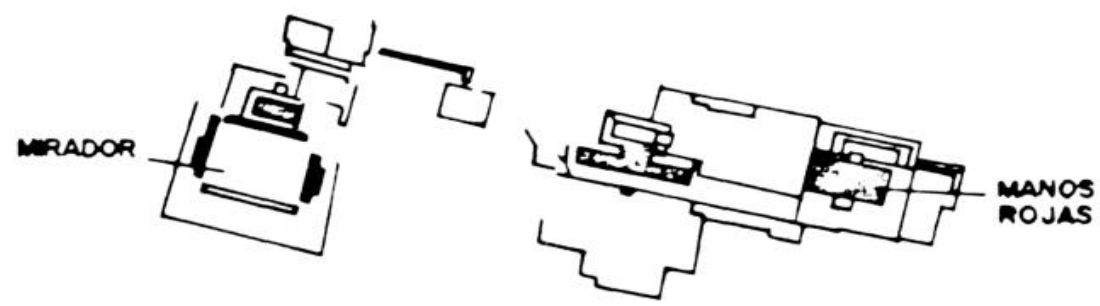

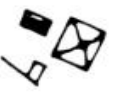
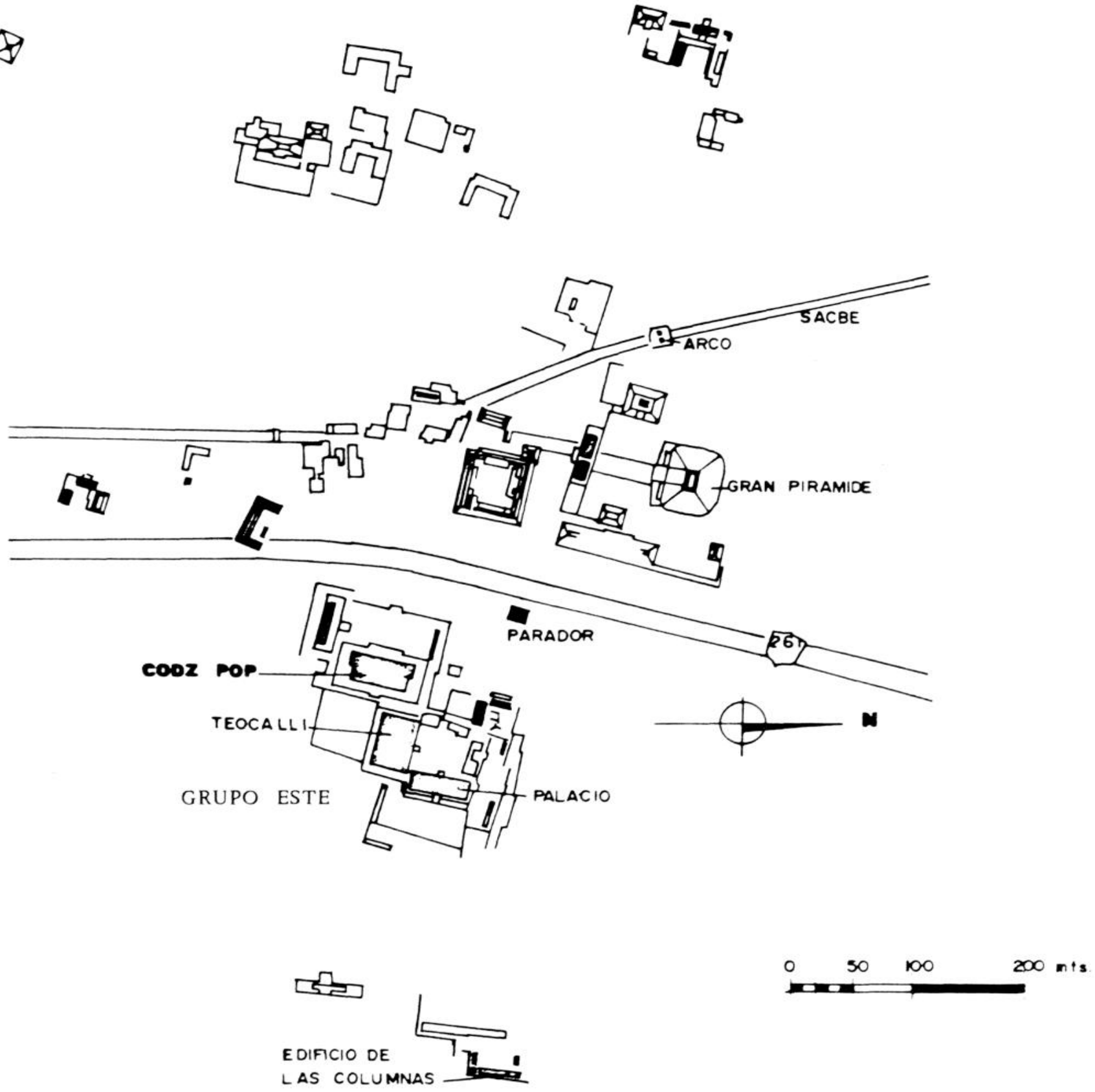

Fig. 2. - Plan général du centre de Kabah, d'après E.M. Shook (P. Gendrop 1977) 


\section{LE GROUPE EST}

Le Groupe Est de Kabah se compose de cinq structures monumentales de nature résidentielle et probablement administrative, de tailles et de proportions différentes (fig. 3). Ces structures bordent la place du Palais (str. 2C2), obtenue par nivellement d'un affleurement rocheux. Stylistiquement, elles correspondent aux différents moments de l'évolution architecturale du site qui couvrent une période débutant par le style Puuc ancien (670-770 ap. J.-C.) et se finissant par le style Uxmal récent (950-1000 ap J.-C.).

En fait, ce groupe est sans doute l'un des plus anciens de Kabah comme l'atteste la présence de céramique préclassique (600-300 av. J.-C.). Dans l'état actuel des recherches, nous ne connaissons pas encore la configuration totale de la place d'origine. Des informations fragmentaires suggèrent qu'une plate-forme rectangulaire supportait des édifices classiques anciens et probablement préclassiques détruits par l'activité architecturale des siècles postérieurs. La plate-forme avait presque acquis sa physionomie actuelle dès son premier nivellement; les modifications apportées par la construction des édifices à travers le temps altérèrent sa taille mais non sa hauteur.

Des cinq structures composant le quadrilatère, quatre ont été explorées. La plus ancienne est la str. $2 \mathrm{Cl}$ ou Groupe du Soleil; elle est suivie, dans la séquence temporelle, par la str. 2C3, ou Teocalli, puis par la str. 2C2, ou Palais, et enfin par la str. $2 \mathrm{C} 4$ qui est postclassique.

La str. $2 \mathrm{Cl}$ a été réalisée dans le style Puuc ancien (670-770) tel qu'il a été défini par Andrews (1986) ${ }^{4}$. Elle est le résultat de cinq phases architecturales successives. Un premier édifice était composé d'une rangée de trois pièces. La pièce centrale s'ouvrait au sud par trois portes séparées par deux colonnes. Elle était flanquée à l'est par une pièce donnant sur la même place et à l'ouest par une autre ouvrant vers le nord, soit à l'arrière de l'édifice. Ce premier édifice était couronné d'ornements ajourés de toiture. Trois pièces furent ajoutées sur son côté ouest. Une de ces pièces prolonge la façade et donne sur la place. Les deux autres furent construites au nord, l'une derrière l'autre, et débouchent sur un patio ouvert sur son côté est. L'ajout d'un troisième bâtiment a complété le patio à l'est. Ce nouveau bâtiment se compose d'une pièce frontale ouverte par un portique à deux colonnes et donnant accès, à l'arrière, à deux petites pièces. Deux rosaces ornent, de chaque côté, les portes communiquant avec les pièces postérieures. Seule la base de la crête faîtière qui repose sur le mur central est conservée. Les quatrième et cinquième phases architecturales n'ont pas été bien définies jusqu'à présent : une pièce prolongeant la façade principale vers l'est a été ajoutée ainsi que deux pièces perpendiculaires, dans l'angle nord-est de l'édifice.

Le style architectural du Teocalli, ou str. $2 \mathrm{C} 3$, se situe à la transition des styles Puuc ancien et à colonnettes. Ce bâtiment peut être considéré comme un bon prototype de l'architecture palatiale Puuc qui se caractérise par l'agencement autour d'une masse centrale de rangs de pièces, longitudinaux et transversaux, 


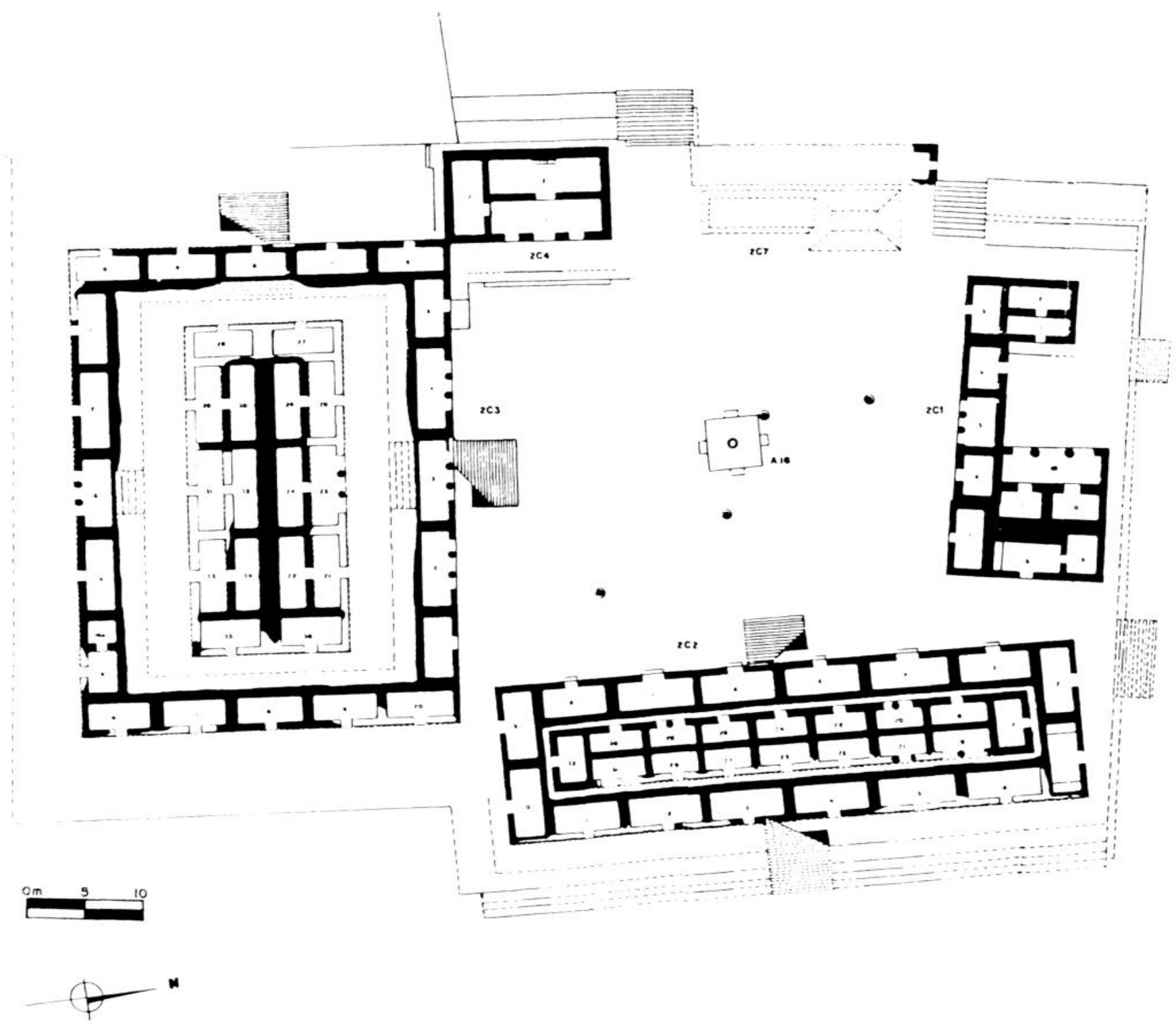

Fig. 3. - Place du Palais ou Groupe Est

simples ou doubles. Cette masse, généralement rectangulaire, sert d'assise à la construction d'un second étage et parfois d'un troisième, comme c'est le cas du Palais de Sayil.

La str. 2C3 fut édifiée en deux étapes. Initialement, cet édifice quadrangulaire n'avait qu'un niveau, l'étage supérieur ne semble pas avoir été envisagé alors. Les pièces sont disposées autour de la masse structurale en une seule rangée. Le décor de la façade est d'une facture très fine et élaborée mais il se rattache encore au style Puuc ancien. Cependant de nouveaux éléments y sont introduits : une double moulure médiane et une moulure supérieure de trois éléments encadrent la frise d'où saillent des supports et des tenons sur lesquels devait s'accrocher une partie 
de la décoration de stuc modelé qui couvrait les quatre façades. Nous sommes intervenus cette saison sur la façade nord. Ses trois pièces centrales s'ouvrent par des portes à deux colonnes qui contrastent avec les accès simples des pièces latérales. Les cinq pièces latérales ouest s'ouvrent chacune par une porte simple et donnent sur une esplanade dont l'accès s'effectuait par un large escalier. Ce dernier fut détruit lors de la construction de la plate-forme du Codz Pop.

Postérieurement, le second étage du Teocalli fut construit ainsi que deux escaliers permettant d'y accéder, l'un adossé à la façade nord et l'autre à la façade ouest. Ces escaliers «volants " forment une espèce d'arc boutant construit selon le principe de la voûte en encorbellement. Ce mode de construction se généralisera par la suite et deviendra presque une exclusivité de l'architecture Puuc.

La structure $2 \mathrm{C} 2$, ou palais, qui donne son nom au groupe, est tout à fait représentative de l'architecture Puuc classique. Contrairement au Teocalli, cet édifice de deux étages fut conçu, dès son origine, dans sa totalité. La maîtrise des techniques de taille et de maçonnerie permit des finitions extrêmement fines tant pour les murs intérieurs que pour les parois externes; on note la généralisation des moulures biseautées, l'introduction d'éléments formels comme la moulure basale en trois parties dont la section inférieure était décorée de colonnettes. De même, les frises de colonnes engagées ou junquillos, lisses ou avec de pseudo-nœuds, alternant avec d'autres élémentş, apparaissent à cette époque. Cela donne à chaque édifice un cachet qui lui est propre à l'intérieur de la tradition Puuc.

Le palais de Kabah est un spécimen de la plus pure architecture Puuc classique. L'architecte a obtenu à la fois solidité structurale et effet de légèreté visuelle en établissant un contraste marqué entre les deux étages et en introduisant une délicate crête faitière ajourée d'au moins trois niveaux. Le parement lisse du niveau inférieur de ce bâtiment qui repose sur un socle de colonnettes est rythmé par trois paires de colonnes engagées et liées. Aux extrémités, ces mêmes colonnes, au nombre de trois, maintiennent la symétrie sur les quatre façades, adoucissent les arêtes du parement inférieur et produisent un contraste de lignes avec le second niveau. La répétition, dans les frises, de l'alternance de panneaux lisses et de colonnes groupées par trois unifie la décoration des deux étages. La crête faîtière, en forme de mur ajouré, est constituée d'une rangée de pseudo-colonnes et d'une rangée de grecques scalaires séparées par une moulure qui rappelle les nœuds des colonnes.

La str. 2C4 est l'édifice le plus récent du groupe : il daterait du début du postclassique. La distribution de ses espaces intérieurs et les caractéristiques de sa façade font de cette structure une construction atypique où les canons de symétrie n'ont pas été respectés. C'est un édifice de trois pièces, deux longitudinales l'une derrière l'autre et une transversale qui s'adosse au coin nord ouest du Teocalli. La porte comporte deux piliers, seul exemple connu jusqu'à maintenant à Kabah. Dans la tradition Puuc, de tels éléments appartiennent à une architecture ancienne, comme c'est le cas de la str. 2C4 de Sayil. Les pierres ayant servi à la construction de la str. $2 \mathrm{C} 4$ de Kabah sont de dimensions variables et leur disposition ne présente pas d'alignements uniformes; elles proviennent sans doute de bâtiments antérieurs ; les moulures supérieures et médianes sont biseautées. Ces diverses caractéristiques distinguent l'architecture récente de l'architecture ancienne dans laquelle les pierres 
sont taillées de façon uniforme, alignées horizontalement et où la moulure médiane est à face(s) verticale(s).

Lors de la construction de la str. 2C4, un édifice antérieur dont nous ne connaissons pas encore les caractéristiques fut détruit, et la plate-forme fut modifiée. Le secteur sud-ouest de la place fut rehaussé de $50 \mathrm{~cm}$, ce qui place la nouvelle structure dans une position plus élevée et marque son importance par rapport aux autres édifices.

Les résultats de cette première saison permettent de proposer quelques conclusions sur l'organisation du Groupe Est et sur son évolution.

On peut considérer qu'une fois les édifices de style ancien (str. $2 \mathrm{Cl}$, str. $2 \mathrm{C} 3$ et d'autres aujourd'hui disparus) construits, l'accès à la place s'effectuait par trois escaliers disposés sur son côté ouest ; un, au nord, était associé à la structure $2 \mathrm{Cl}$; un autre était situé au centre et le troisième, au sud, menait au Teocalli. Postérieurement, l'escalier sud fut presque totalement démonté et recouvert par la grande plate-forme du Codz Pop. L'escalier central fut également démonté pour être remplacé par un escalier plus élevé lors de la construction de la str. $2 \mathrm{C} 4$ et de l'agrandissement de la plate-forme.

Le Groupe Est est un élément essentiel à la compréhension de l'évolution de l'architecture de Kabah et de la région Puuc en général. C'est un exemple rare réunissant en un seul contexte plusieurs styles architecturaux qui se sont succédé dans les Basses Terres mayas septentrionales. Cette première incursion dans l'archéologie de Kabah nous a permis d'approcher, à travers son architecture, une partie de son évolution historique. La fouille plus détaillée de certains secteurs du Groupe Est et celle d'autres groupes sont nécessaires pour compléter les informations disponibles et élargir nos connaissances sur l'occupation du site et son évolution.

\section{LE CODZ POP OU STR. 2C6}

Le Codz Pop de Kabah se situe au sud-ouest du Groupe Est. Bien qu'inséparable du Groupe Est, il fut construit sur sa propre plate-forme qui s'élève de trois mètres au dessus de la place du Palais et fait de lui un élément urbanistique indépendant. Cette construction monumentale d'un étage a un plan en forme de $\mathrm{T}$ ou $i k$ (fig. 4). Les façades ouest et est mesurent respectivement $54 \mathrm{~m}$ et $46 \mathrm{~m}$, ses côtés nord et sud ont une longueur de $24,5 \mathrm{~m}$. L'édifice se compose, à l'ouest, de deux rangées parallèles de cinq pièces et, à l'est, d'une rangée simple de neuf pièces; au nord et au sud, il existe deux pièces alignées et deux autres en enfilade.

Deux accès conduisent au Codz Pop. A l'ouest un premier escalier mène à l'esplanade où se trouvent un autel hiéroglyphique et le chultún (citerne souterraine). De là, un second escalier faisant face à la façade des masques de chacs, mène au Codz Pop. L'accès à la plate-forme par l'escalier est n'a pas encore été exploré.

Le côté est de la plate-forme du Codz. Pop se compose de trois corps et les autres côtés de deux. Une tranchée a dégagé une partie de cette plate-forme et mis 


\section{Illustration non autorisée à la diffusion}

Fig. 4. - Le Codz. Pop ou Str. 2C6 (complété par J. Ligorred d'après Pollock [1980])

au jour des éléments de la décoration. Il s'agit, probablement, de la décoration que Pollock (1980) localisa à l'angle sud-est du Codz Pop et qui formerait le troisième corps de la plate-forme. Le dégagement de ces corps permettrait une étude détaillée de leurs caractéristiques morphologiques.

Les nouvelles informations recueillies suggèrent que l'architecture de l'édifice se rattache au style Uxmal récent (950-1000) bien que beaucoup d'auteurs la placent dans le style Mosaïque (800-830). La façade ouest, déjà dégagée, a été décrite par Gendrop (1983:41) ${ }^{5}$ en ces termes : "cas extrême de l'architecture Puuc, cette construction est entièrement couverte de sculptures d'un relief prononcé, dont le fini justifie pleinement le nom de Kabah, qui signifie selon certains auteurs la main qui cisèle ". Ces sculptures couvrent totalement la façade ouest. Elles représentent des masques de la divinité au nez recourbé connue sous le nom de Chac.

Des tranchées successives de $2 \mathrm{~m}$ de large ont libéré des déblais le centre de la façade est sur 18 mètres, au niveau des pièces 20,21 et 22 . Elles ont mis au jour, sur le sol de la plate-forme, la décoration qui ornait la frise et la moulure médiane de la façade et elles ont dégagé une grande partie du parement inférieur en place. 
Le mur est décoré de panneaux croisillons séparés par d'autres, à trois éléments verticaux accolés : de chaque côté, un empilement de triangles rectangles superposés, base contre pointe, et, au centre, un entrelacs vertical. Le fond était recouvert d'une couche de stuc peinte en rouge qui estompait l'aspect de mosaïque de pierres que nous observons à présent.

Une épaisse moulure médiane reposait sur le mur inférieur. Elle était formée par trois éléments : une grande corniche ornée d'une frange de plumes et de fleurs en relief, un corps de serpent composé d'un décor en dent de scie et de petits triangles et une corniche supérieure décorée de bandes entrelacées.

Sur la frise ornée de grandes nattes, sept sculptures monumentales du Roi de Kabah se dressaient avec autorité (fig. 5). Deux têtes de ces sculptures avaient déjà été découvertes ${ }^{6}$ et le Roi de Kabah avait été identifié auparavant par sa face scarifiée, ses cheveux tressés et sa coiffe enroulée, nouée par une grande attache anthropomorphe. Les derniers travaux à Kabah ont permis de compléter ces sculptures par la découverte des corps et des grandes coiffes de plumes. Les personnages ont une hauteur de $2 \mathrm{~m}$ et chaque module couvre une largeur de 2,25 $\mathrm{m}$. Les trois éléments composant le personnage - tête, tronc et bras ; la ceinture ; les extrémités inférieures - sont sculptés en ronde bosse. Ces sculptures faisaient saillie du bas relief les entourant qui, par une mosaïque de pierres taillées, représentait la coiffure de plumes. Au-dessus de la tête du personnage, comme s'il s'agissait de la coiffe, un grand masque d'une divinité zoomorphe au nez crochu semblable aux Chacs de la façade ouest est taillé en un seul bloc et scellé dans le mur par une tenon épais.

La partie supérieure de la frise, les limites de la coiffure et la moulure supérieure n'ont pas été localisées pour le moment. La hauteur de la façade étant supérieure à la largeur du dernier niveau de la plate-forme, les pierres les plus hautes sont certainement tombées dans des niveaux bas qui n'ont pas encore été fouillés.

Bien que découverts il y a des années, les jambages de la porte de la pièce 21 étaient demeurés sous les décombres. Le nettoyage de ce secteur a permis de les dégager à nouveau, d'apprécier et d'enregistrer leurs caractéristiques. Chaque jambage se compose de deux grands blocs ornés chacun d'une scène taillée en haut et bas relief. Ainsi, quatre scènes apparaissent sur ces deux jambages en une parfaite symétrie. Deux personnages dansant, probablement dans un rituel de guerre, sont représentés dans la partie supérieure des jambages. Il est important de souligner la présence de l'Atlat, instrument de guerre normalement associé à l'arrivée des Itzas. Un dieu zoomorphe se distingue en fond. Son profil est typique des derniers moments du style Puuc. Une bande de glyphes sépare les scènes supérieures et inférieures. Les scènes inférieures représentent une capture : le roi de Kabah saisit par les cheveux un prisonnier agenouillé à ses pieds, un troisième individu, les armes levées, célèbre probablement un triomphe.

L'édifice est couronné d'une grande crête faîtière ajourée de type "péninsulaire » (Gendrop 1983 : 187). Il s'agit d'une superstructure simple rythmiquement ajourée, reposant sur le mur postérieur de la rangée interne des pièces ouest et sur la masse structurale de l'édifice. Il a été possible de restituer un second niveau formé par la répétition de grecques scalaires. La disposition des décombres et des grecques scalaires qui y sont conservées permet de supposer que la crête possédait 


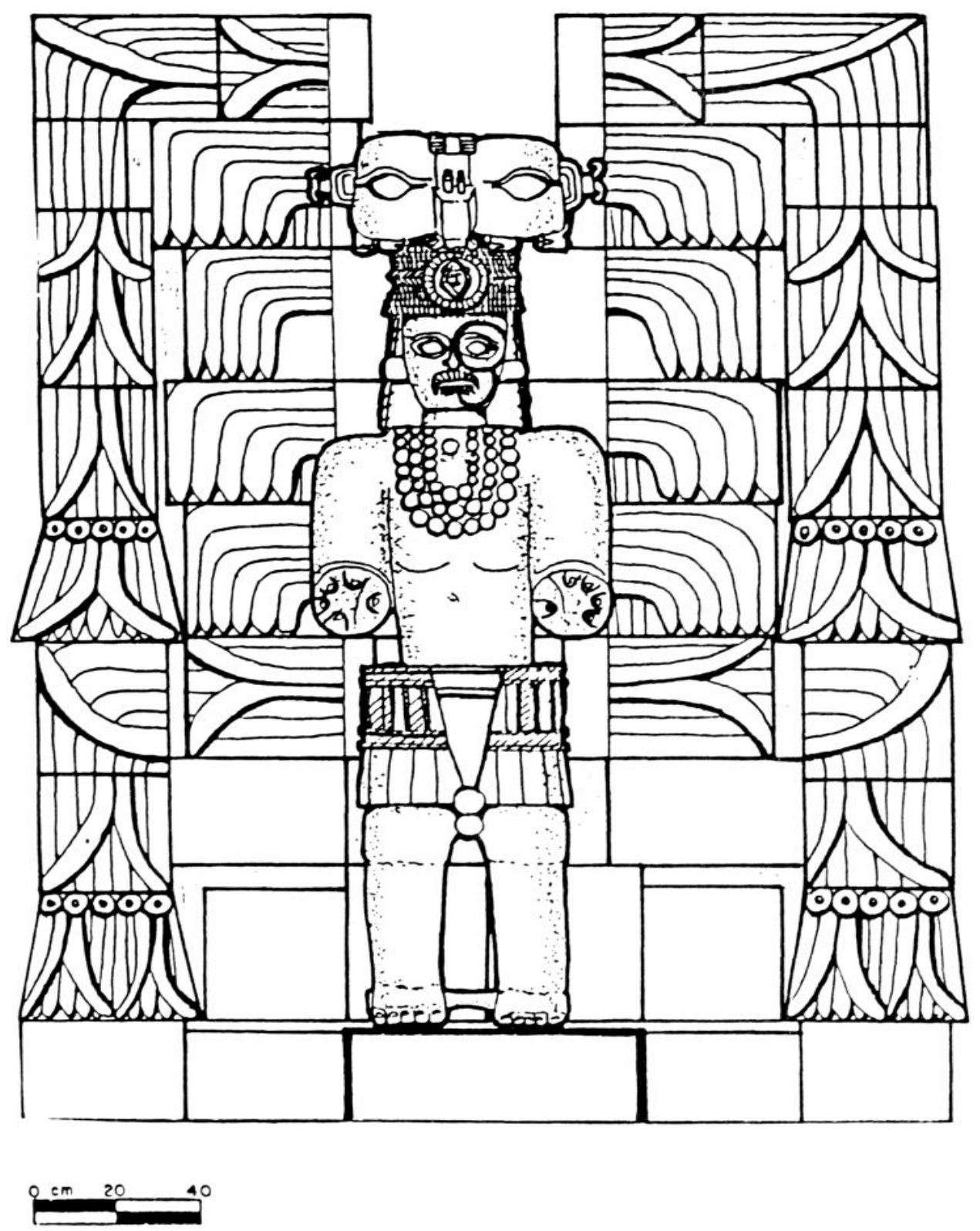

FIG. 5. -- Le « Roi de Kabah » (Codz Pop, façade est).

deux niveaux supplémentaires : un troisième, également de grecques, et un quatrième, fait probablement de colonnes et donc semblable au premier niveau.

Ainsi, l'exemple du Codz Pop permet d'approcher la conception esthétique de l'architecture classique du Puuc : l'intense charge iconographique des façades, avec tout son baroquisme, se résout en une fine prolongation couronnant la structure.

Des découvertes d'une toute autre nature révèlent des faits historiques primordiaux qui, à leur tour, valorisent la signification des masques des dieux zoomorphes couvrant la façade ouest du Codz Pop. Sous le sol de la pièce 8, au centre de la rangée occidentale postérieure, il existe une sépulture voûtée qui fut rituellement violée probablement au Postclassique. 
Presque sur le sol de la pièce 8 , une épaisse couche de charbon et de cendres est apparue, associée à des fragments d'encensoirs polychromes postclassiques du complexe Hocaba (1200-1300 ap. J.-C.). Immédiatement sous cette couche, on a découvert des fragments de nez ainsi que d'autres parties qui composaient les masques de la façade et des dalles de voûtes. La position des pièces suggère qu'elles auraient été placées à cet endroit lors d'un rituel en liaison avec la dernière occupation du site.

Dans une couche inférieure, des os humains sont apparus épars et sans connexion anatomique. Quatorze pièces céramiques fragmentées, un pendentif en cristal de roche et un fragment de couteau de silex ont dû faire partie de l'offrande accompagnant le mort. De petits fragments de plaques roses, portant des traces de polychromie, auraient été le revêtement d'un objet de bois, d'après les empreintes notées sur leur côté postérieur. Une analyse préliminaire des quatorze céramiques fragmentées situe l'enterrement dans l'horizon Cehpech du Classique Final, entre 750 et 1000 de notre ère.

Une première interprétation est possible : la tombe voûtée, selon sa facture, ses dimensions, sa localisation et l'offrande qui lui est associée, aurait abrité un personnage important de Kabah. Elle aurait été violée postérieurement et des fragments de masques y auraient été déposés. Certains, plus larges et plus grands, auraient appartenu aux chacs des entrées. Les traces de peinture observées sur certains fragments suggèrent qu'ils furent enterrés immédiatement après avoir été décrochés de la façade. Le charbon, les cendres et les encensoirs permettent de penser qu'un rituel se serait déroulé durant le postclassique.

\section{MANOS ROJAS OU STR. 1A1}

La str. 1Al de Kabah, connue également sous les noms de Manos Rojas ou Dzalkabilkik (en maya, " les empreintes des mains de sang ») et d'Édifice au linteau de bois taillé, se trouve dans le Groupe Ouest, au nord du Mirador et du Temple des Linteaux. De plan général rectangulaire, elle comporte deux étages correspondant à deux phases architecturales successives. Le premier niveau, composé de pièces alignées sur les quatre côtés, se caractérise par la décoration de style mosaïque de sa façade est. L'étage supérieur est un temple à entrée zoomorphe divisé en deux pièces (21 et 22). Dans l'attente de données de fouilles supplémentaires, nous distinguerons seulement deux phases de construction; nous jugeons les données visibles insuffisantes pour en distinguer davantage comme le fait Pollock.

Les travaux menés récemment ont permis l'exploration et le dégagement de l'escalier est, d'une partie de la façade occidentale correspondant aux pièces 4,5 et 6 et de l'intérieur des deux premières. Cette partie du bâtiment fut également restaurée. Ces fouilles mirent au jour la première marche de l'escalier est, formant la mandibule inférieure de l'entrée zoomorphe. L'accès à la pièce 6 apparut sous la forme d'une banquette composée de deux marches, bordée de petites colonnes sur ses trois côtés visibles. Cinq pierres taillées en forme de tête de mort furent découvertes dans les décombres de la façade; elles devaient être encastrées dans la 
frise de grecques et de colonnes par leurs tenons. Un fragment de panneau comportant les huit glyphes, encore lisibles, qui terminent une inscription fut également trouvé. Au niveau de la pièce 6 sont apparus les vestiges d'un muret de pierres accolés au bâtiment. Ce muret est de facture grossière mais a été construit avec des pierres taillées, certaines décorées provenant de la façade ; il atteste d'une réoccupation postclassique.

Nous devons à Stephens et Catherwood (1986 : II : 345) ${ }^{7}$ la première description de ce bâtiment et de son linteau de bois à présent disparu :

"Au nord ... il y a un autre édifice de 142 pieds de long et 31 de large, ses doubles couloirs
correspondent entre eux et un gigantesque escalier, en son centre, mène au toit sur lequel
se remarquent les ruines d'un autre édifice. Tous les linteaux des portes sont en bois, en
place et pour la plupart en bon état. Les portes étaient obstruées de terre et de décombres,
M. Catherwood a dû franchir la porte la plus proche de l'escalier, bouchée jusqu'à trois
pieds de son linteau, en rampant sur le dos, pour prendre les dimensions intérieures d'une
pièce. Une fois dans la pièce, un linteau gravé attira son attention; après l'avoir examiné,
il jugea que cet objet était le plus intéressant que nous ayons trouvé au Yucatán. Je le vis
le jour suivant et décidai aussitôt, qu'à n'importe quel prix, je l'emmènerais dans mon
pays. "

En effet, Stephens détacha le linteau et l'emporta à New York où il disparut lors d'un incendie. Ce linteau était composé de deux poutres, il représentait un souverain debout et de face dont la tête était tournée vers la droite. Son style, selon Proskouriakoff $(1950: 167)^{8}$, " n'est pas plus ancien que la date 9.18.0.0.0 et date probablement du cycle 10 », ce qui le place entre 790 et 830 de notre ère.

Cette sculpture est particulièrement remarquable par l'ornementation du personnage (sans doute celui qui commanda la construction du bâtiment). Il porte une grande coiffure de plumes avec un masque probablement zoomorphe. Son vêtement est également luxueux, orné d'un grand pectoral et d'une ceinture. Il présente une bourse dans la main gauche et de l'autre main, un sceptre-figurine, probablement du dieu $\mathrm{K}$. Malheureusement, le visage et la coiffe étaient très détériorés. Dans les sites mayas, les représentations de souverains exhibant un sceptre-figurine sont associées au thème de l'ascension au pouvoir.

Les impressions murales des mains rouges furent également remarquées par Stephens.

"Encore une fois, nous avons rencontré l'impression de mains rouges, pas une ou deux, ou trois comme en d'autres lieu; tout le mur était couvert de ces mains rouges, claires, brillantes comme si elles venaient d'être refaites. » (ibid.)

L'impression de mains est une manifestation picturale rituelle très universelle. Dans le cas du Yucatán septentrional, les impressions de mains apparaissent dans quelques grottes et certaines constructions possédant, en particulier, des portes zoomorphes, transpositions symboliques de l'entrée dans les grottes (c'est-à-dire dans l'inframonde). Très répandues, elles se rencontrent, sur stuc ou sur pierre, dans des édifices de Kabah, Uxmal, Chichén Itzá, Cobá, Tulum... D’après leur taille (entre 12 et $13 \mathrm{~cm}$ ) des mains d'enfants ont dû servir à décorer les parois de la structure 1A1. A l'exception d'un petit alignement, elles ne suivent pas un ordre 
discernable. Ces mains, associées à l'entrée zoomorphe et donc au caractère chthonien de l'édifice, permettent d'émettre l'hypothèse suivante : lors d'un rituel ayant rapport avec la pluie, des enfants auraient été tenus d'imprimer leurs mains. Sans grand risque d'erreur, nous pouvons attribuer ce rituel à l'époque même de la construction du monument; en effet la détérioration a mis au jour les mêmes traces de pigment sur la couche préalable de stuc. Cela signifie que, dès leur conception, les pièces 4 et 5 étaient destinées à servir d'espace où aurait été célébré le rituel d'impression de mains. Des dessins géométriques sont également visibles mais leur signification est incertaine. Leur facture grossière indique qu'ils furent réalisés avec les doigts.

L'entrée zoomorphe, œuvre de très grande qualité totalement taillée dans la pierre, est un autre élément important, aujourd'hui hélas très détruit. Bien que plus élaborée, elle montre de grandes similitudes avec celle du temple IV ou du Devin de Uxmal. Il semble qu'elle se rattache au style Uxmal Récent. Deux tentatives de restitution partielle furent réalisées par Pollock (1980, fig. 280). Cette porte offre les bases permettant de considérer les pièces 21 et 22 comme un espace rituel, probablement un temple. Par son rôle de grotte, ce temple représenterait le seuil conduisant à l'inframonde, la grotte étant le lieu où se manifestent les divinités et le point de communication avec le monde surnaturel.

En conclusion, nous considérons que les éléments signalés ci-dessus constituent une base suffisante pour attribuer à la structure $1 \mathrm{Al}$ une fonction cérémonielle et religieuse. Le symbolisme de la grotte, dont elle paraît chargée, est à rapprocher du concept de montagne sacrée qui apparaît dès le classique ancien (Schele et Freidel 1990) ${ }^{9}$. Ces caractéristiques rituelles et cérémonielles distinguent l'édifice (et sans doute l'ensemble du Groupe Ouest) des autres zones du site, comme le Groupe Est, dont les fonctions auraient été surtout politiques et administratives.

\section{UNE OCCUPATION POSTClASSIQUE :}

Tout le long de l'édifice et sur l'ensemble de sa plate-forme, une réoccupation tardive a été notée, comme dans d'autres secteurs de Kabah (Teocalli, Codz Pop et str. 2C4). A Manos Rojas, elle se manifeste par l'emploi de pierres taillées provenant de l'édifice et de l'entrée zoomorphe dans la construction de structures domestiques. Elle a ainsi contribué à la détérioration du bâtiment.

Les vestiges de cette réoccupation ont été découverts sous les décombres de la façade est et fouillés. Ce sont des alignements de pierres, des espaces dallés et les restes d'un muret de pierres sèches construit autour de la banquette de la pièce 6 . Les fouilles ont mis au jour trois foyers, deux décharges, plusieurs meules et de nombreux instruments lithiques (une hache, un ciseau, des molettes, des polissoirs et des pilons) et de la céramique. Selon une analyse préliminaire de la céramique, les résultats des datations ${ }^{14} \mathrm{C}$ n'étant pas encore disponibles, nous supposons que ces vestiges correspondent à une assez longue occupation postclassique que prolonge l'horizon historique de Kabah. Ces nouvelles données soulèvent de nombreuses questions à propos du postclassique du nord du Yucatán. Smith avait déjà enregistré du matériel Sotuta et Hocaba à Kabah mais son échantillon était très faible : exactement deux tessons pour chaque phase. Avec la réoccupation de 
Manos Rojas c'est tout un espace qui appartient au postclassique.

En résumé, et dans l'attente des résultats définitifs des analyses du matériel et des échantillons collectés, nous envisageons la réoccupation postclassique comme un établissement étendu le long de l'édifice ancien, sous la forme de maisons en matériaux périssables, les pièces originales du bâtiment étant elles-mêmes réutilisées à des fins domestiques.

Pour le moment, nous pouvons distinguer deux aires d'activité principales. La première, dans le couloir et face aux pièces 4 et 5 , se caractérise par deux foyers, des alignements de pierres, un matériel culturel abondant et des os d'animaux (il est intéressant de souligner également la découverte de petites boules ou billes d'argile, dont la fonction demeure incomprise). L'autre aire importante est centrée autour de la banquette en escalier de la pièce 6 . Elle est associée à une meule complète et à un foyer; le muret qui l'entoure suggère la délimitation de plusieurs aires à l'intérieur de l'édifice.

\section{LA STRUCTURE DE L'HABITAT ET LES LIMITES DE KABAH}

Si l'essentiel des travaux, durant la saison 1991, fut concentré sur l'exploration et la restauration de quelques grands édifices, les recherches concernant la structure de l'habitat n'en sont pas moins importantes. Le premier objectif a été de compléter le plan établi par Pollock (1980).

C'est dans cette optique qu'une première reconnaissance de surface a débuté en 1991 dans le secteur occidental du site; elle sera étendue aux autres secteurs postérieurement. Elle a permis de tracer les limites occidentales du site et de découvrir de nouveaux groupes dont certains, présentés ici, sont d'importance majeure.

Une première brèche, de $1500 \mathrm{~m}$, a été tracée vers l'ouest, à partir du Mirador (str. 2A1). Un groupe de plates-formes domestiques représente les dernières structures localisées le long de cette brèche; elles se trouvent sur le flanc d'une colline calcaire (huitz), à $900 \mathrm{~m}$ du Mirador. Au-delà de la colline, sur les $600 \mathrm{~m}$ restant, s'étend une plaine sans vestige archéologique. Du point extrême de cette première brèche, on a réalisé un parcours selon un axe perpendiculaire de $1000 \mathrm{~m}$ vers le sud. Durant ce trajet, aucune plate-forme n'a été localisée. Les autres points proposés comme limites du site se trouvent sur la route, au sud, à $1700 \mathrm{~m}$ de l'entrée du site et, au nord, à $1400 \mathrm{~m}$ Un dernier point se situe sur le chemin menant du point nord, vers l'ouest, à l'unité agricole Felipe Carrillo Puerto, à une distance de $1800 \mathrm{~m}$; à $800 \mathrm{~m}$, nous avons croisé le sacbé (chaussée surélevée) menant à Nohpat puis à Uxmal.

Ainsi, lors de cette première saison, quatre points principaux ont été fixés pour l'étude des limites du site : les points sud et nord, le long de la route; les points ouest à $1500 \mathrm{~m}$ du Mirador et à $1800 \mathrm{~m}$ du point nord.

L'aire parcourue présente une succession de collines calcaires plus ou moins élevées (huitz) et de plaines (planadas ou cancabales). Les collines ont souvent été mises a profit par les anciens mayas tant pour la construction des plates-formes des 
grands groupes cérémoniels que pour celle des unités domestiques.

Six nouveaux groupes ont été localisés durant cette première reconnaissance.

Groupe I :

Ce groupe, construit sur une élévation naturelle, se trouve à $1200 \mathrm{~m}$ au nord-ouest du Codz Pop. Huit édifices voûtés et six plates-formes ayant supporté des structures construites en matériaux périssables sont répartis sur quatre places. Cinq édifices ont conservé une partie de leur voûte et trois sont totalement en ruines. On peut observer des colonnes et des jambages soutenant des linteaux encore en place.

La place 1, ou place principale, est un quadrilatère bordé par des structures voûtées; deux chultunes ont été localisés, un dans l'angle nord-est de la place et l'autre, à l'ouest de la structure nord. La place 2 est ouverte au nord et fermée par des édifices voûtés sur les autres côtés. Les côtés nord, est et ouest de la place 3 sont occupés par des plates-formes ayant supporté chacune deux structures en matériaux périssables. Trois structures de même type reposaient sur une plate-forme au sud de la place 4 ; face à cette plate-forme, un grand espace avait été laissé vide.

\section{Groupe II :}

Situé à $1000 \mathrm{~m}$ du Codz Pop, il fut également construit à partir d'une élévation naturelle artificiellement nivelée. Des vestiges de voûtes, de jambages et de colonnes y sont visibles ainsi que des terrasses destinées à des structures en matériaux périssables et des chultunes.

\section{Groupe III :}

Le groupe III se trouve à $400 \mathrm{~m}$ au sud-ouest du Codz Pop. Une plate-forme artificielle a été construite sur un terrain plat. Deux structures atteignent une hauteur considérable et, parmi les pierres éboulées qui les entourent, se trouvent des éléments de masques. Ces édifices sont accompagnés d'autres structures voûtées.

\section{GROUPE IV :}

Situé à $550 \mathrm{~m}$ au sud du Codz Pop, le groupe IV fut édifié sur le flanc d'une colline. Des structures ont été construites à sa base, au nord; la pente elle-même présente plusieurs nivellements artificiels et l'on y a aménagé des escaliers et d'autres bâtiments. Au sommet de la colline, au sud, il existe une structure pyramidale aujourd'hui effondrée. Face à elle, un espace de $35 \mathrm{~m}$ a dû tenir lieu de place ou d'aire d'activité rituelle. Dans ce groupe, comme dans les autres, des chultunes sont visibles. 
GroUPE V :

Le groupe V, à $550 \mathrm{~m}$ au nord du Codz Pop, repose sur une élévation naturelle. Cinq structures effondrées ont été localisées. Du fait de sa position en bordure de la route fédérale, l'élévation sur laquelle fut construit le groupe a été arasée sur son côté ouest.

\section{Groupe VI :}

A $800 \mathrm{~m}$ au nord du Codz Pop, ce groupe se situe dans les terres du rancho Santa Ana. Une plate-forme artificielle s'élève sur un terrain plat. Les édifices sont voûtés et, par endroit, des voûtes et des jambages sont préservés. Une des pièces a conservé presque toute sa voûte.

\section{LA PLATE-FORME DU CABRIO}

La plate-forme du Cabrio a été l'habitation de gens humbles de Kabah et le siège de leur vie domestique. Par des fouilles intensives nous avons tenté d'identifier les fonctions des bâtiments et d'éventuelles aires d'activité. En présentant ici quelques résultats préliminaires, nous voudrions souligner l'importance de ce genre de fouilles trop souvent négligé dans les basses terres mayas au profit d'une approche globale de l'habitat. Se limiter à une description de surface engendre des risques d'erreur ou, au moins, laisse dans l'ombre des informations importantes.

Le groupe du Cabrio a été découvert par Ramón Carrasco ${ }^{10}$ lors de la reconnaissance du sacbé reliant Kabah à Nohpat puis à Uxmal. Il se situe à $750 \mathrm{~m}$ de l'arc de Kabah et se compose d'une structure pyramidale et de deux plates-formes d'habitation de chaque côté du sacbé. Seule, la plate-forme du Cabrio (fig. 6) située à l'ouest du sacbé a été fouillée. Les informations issues de la prospection suggéraient que cette plate-forme était une unité domestique composée de logis et d'annexes ayant abrité une famille étendue. La description de surface après le défrichement nuança cette vision : au sommet même de la plate-forme, on pouvait observer un chultún, son aire de captage et les vestiges de quatre bâtiments (str. I, II, III et VI). Après les fouilles, ce sont au total huit structures qui ont été relevées sur un espace de $730 \mathrm{~m} 2$. La dernière occupation du groupe date de l'horizon Cehpech (750-1000 ap. J.-C.). Mais il y a des traces d'occupations antérieures avec de la céramique pizarra pre-Cehpeh (600-750) et d'autres types appartenant au Classique Récent et au Préclassique.

La fouille a mis en évidence que les $730 \mathrm{~m}^{2}$ se divisaient en trois sections morphologiquement différentes, deux sur la plate-forme, l'une stuquée l'autre non, et la troisième aux abords immédiats (fig 6). Les quatre bâtiments de la zone stuquée étaient construits selon le même principe. Il ne reste que le assises en pierres des murs, leur partie supérieure et les toits étant faits de matériaux périssables, le sol était stuqué. Des différences de dimensions, de facture ainsi que la céramique 


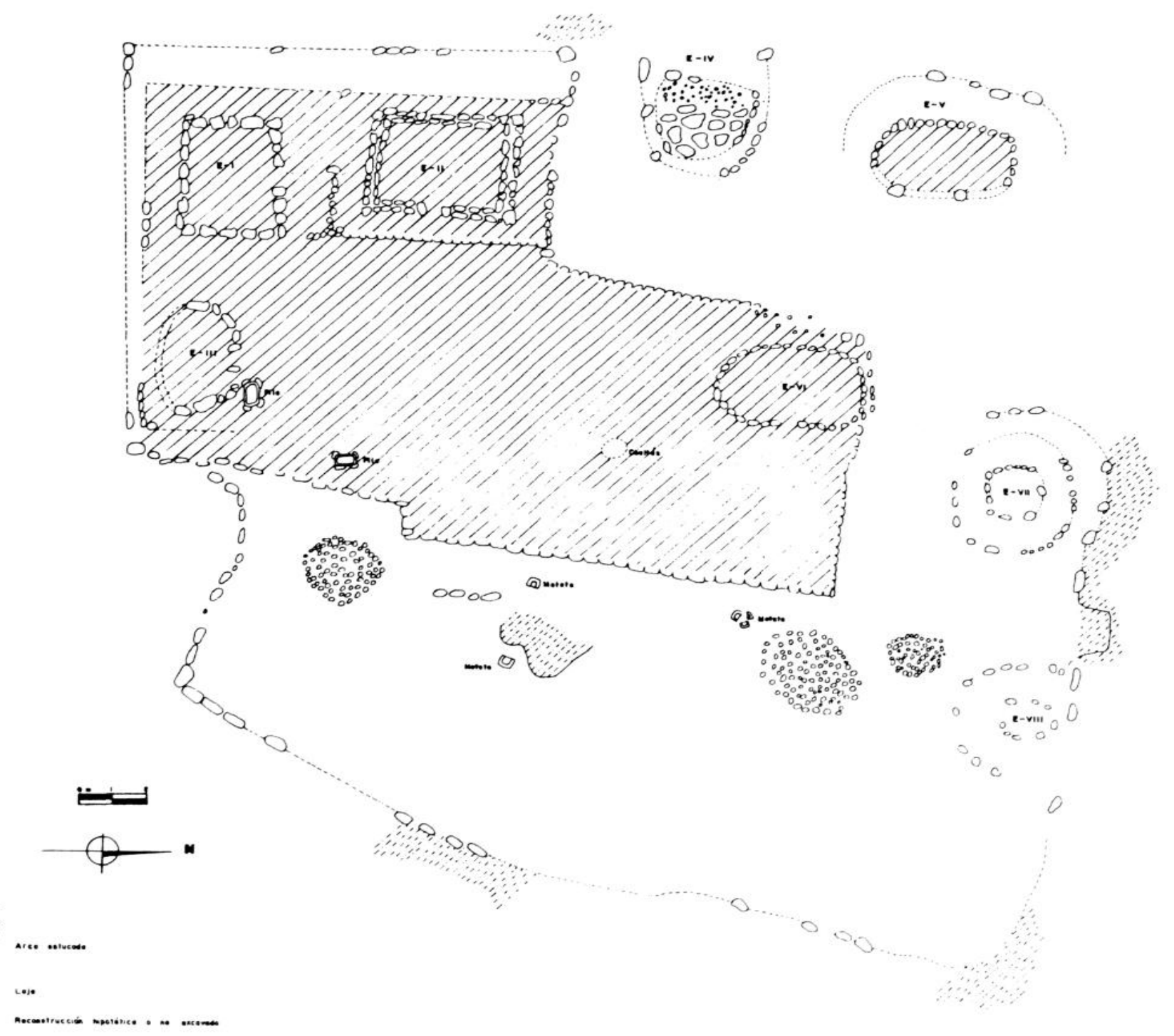

Fig. 6. - La plate-forme du Cabrio

associée suggèrent des variations fonctionnelles. Une fois fouillés, quatre monticules de pierres qui semblaient identiques en suface (IV, V, VII, VIII) sont apparus de nature différente.

Dans cette région démunie de sources, l'espace domestique est en grande partie déterminé par la nécessité de capter les eaux de pluie et de les diriger vers une citerne souterraine, le chultún. La section stuquée de la plate-forme est une aire de captage des eaux de pluie. Le chultuin de la plate-forme du Cabrio aurait eu une profondeur de $4 \mathrm{~m}$. Une fois le chultún creusé, l'élévation initiale aurait été égalisée par l'ajout d'un remblai de pierres retenu par le mur de contention de la plate-forme. L'aire de captage a été délimitée précisément au nord et à l'est. Ailleurs, elle rejoint le mur de contention. Sa surface a été stuquée de telle sorte qu'une légère dénivelée facilitât l'écoulement des eaux de pluie vers le chultún. Ce n'est qu'une fois cet ensemble aménagé que les bâtiments furent construits. 
La str. II est le bâtiment le plus grand $\left(10 \mathrm{~m}^{2}\right)$; elle est la seule à avoir été construite sur une terrasse et à posséder un soubassement en maçonnerie d'au moins $80 \mathrm{~cm}$. Ces caractéristiques suggèrent qu'il s'est agi du le bâtiment principal. Sur le sol, une couche de tessons indique que cette structure a été abandonnée durant l'horizon Cehpech. Le long des murs du bâtiment, on a recueilli des vestiges de jarres striées et de bassins, preuve qu'ils étaient brisés sur place, adossés contre les parois. Au centre de la structure, les tessons sont beaucoup plus abondants. Nous avons estimé qu'ils représentaient un plat pizarra, un pot et sept jarres striées destinées à emmagasiner l'eau. Comment expliquer leur présence en aussi grand nombre au centre du bâtiment? On peut certes imaginer que le bâtiment a eu d'autres fonctions que résidentielles; mais on peut aussi penser que les jarres y étaient simplement stockées, suspendues ou rangées sur les poutres de la charpente, par exemple. La disposition des tessons paraît confirmer cette hypothèse, aucun fond n'ayant été retrouvé incrusté dans le sol.

La str. I, à moins de $8 \mathrm{~m}$ de distance, est moins élaborée. Son soubassement de pierres sèches équarries ne dépasse pas $60 \mathrm{~cm}$ de hauteur. La présence d'un foyer a été déduite des pierres brûlées du remblai et de la cendre associée. La céramique provenant de cette structure appartient également à l'horizon Cehpech et les formes majoritaires (bassins, plats et bols) ne contredisent pas, au contraire, l'hypothèse qu'il s'agirait d'une cuisine, suggérée par la présence d'un foyer, le seul trouvé sur l'ensemble de la plate-forme.

La str. III, de forme elliptique, est de facture encore plus grossière. Sa fonction n'a pas été établie mais il semble que des activités pour lesquelles le maniement de l'eau était important s'y soient déroulées. La proportion de tessons de jarres striées est exceptionnellent élevée. Certains étaient associés à quatre pierres intentionnellement calées qui ont dû supporter une de ces jarres. A l'extérieur, une meule devenue trop profonde par son utilisation répétée (nommée dans ce cas pila) a changé de fonction et fut placée sous un pan du toit pour recueillir l'eau de pluie. Peut être faut-il associer cette pila à la pierre à moudre à peine entamée située à moins de $6 \mathrm{~m}$. Dans ce cas, la str. III pourrait être une réserve où on entreposait le maïs.

La fonction de la str.VI, également de facture grossière et elliptique, n'a pas été identifiée. L'abondance des tessons de jarres striées et son ouverture dirigée vers le chultún situé à moins de $6 \mathrm{~m}$ suggèrent encore un lien avec l'eau.

Bien que les fonctions des bâtiments ne soient pas encore bien établies, il apparaît clairement que cette section stuquée était à la fois un dispositif hydraulique et un espace où se déroulait une grande partie des activités purement domestiques - au moins celles nécessitant un abri. L'aire de captage des eaux de pluie devait rester propre, aussi peut-on s'étonner que quatre bâtiments y aient été construits. Mais les bâtiments ainsi situés ont le double avantage d'être proches de la source d'eau et de compléter le captage des eaux de pluie par leurs toits. L'eau était un souci primordial des anciens habitants de la région Puuc. Nous avons vu qu'une pila avait été disposée sous les pans du toit de la str. III; nous avons également trouvé le long d'un des murs latéraux des str. I et II, à proximité des bords de la plate-forme, des concentrations de tessons de jarres striées. Elles étaient probablement disposées à cet endroit pour recueillir l'eau de pluie et faciliter son 
utilisation sans contaminer l'aire de captage. A l'extérieur de la plate-forme, la plus forte densité de tessons se trouve au sud de la section stuquée; on peut penser qu'on a à faire à cet endroit à la décharge.

La nature des deux autres espaces n'a pas encore été bien définie. A l'est de l'aire de captage s'étend la section de plate-forme qui n'a pas été stuquée. De petites concentrations de pierres y furent remarquées ainsi que deux pilas. Il serait logique de penser que cet espace était destiné aux tâches domestiques quotidiennes réalisées à l'extérieur de l'aire de captage. Mais cette vision est peut-être contredite par la présence des structures VII et VIII. Il s'agit de petites élévations circulaires ressemblant fortement aux autels des zones monumentales. Dans le remblai de la str. VII, les sépultures de deux individus ont été mises au jour; un plat et un vase de l'horizon Cehpech accompagnaient les morts ainsi qu'un plat pre-Cehpech. Sur cette structure, un nombre important de tessons de céramique (1070) a été collecté.

Aux abords immédiats de la plate-forme, les structures IV et $\mathrm{V}$, identiques avant la fouille, se sont révélées totalement différentes une fois fouillées. La str. IV, semi-circulaire, est bordée par une rangée de pierres. A l'intérieur, la moitié ouest est recouverte de petites pierres. Le sol de la moitié est, qui est plus basse, est fait de grandes pierres plates et est bordé d'une marche. Il était recouvert d'une couche de cendre et de pierres brûlées. Un nombre considérable de tessons est issu de cette structure ( 3727 , soit $34 \%$ de la collection du groupe) et en particulier de sa moitié est. La comparaison avec d'autres collections permettra peut-être de préciser si certaines anomalies notées sur cet échantillon sont liées à la cuisson et de vérifier l'hypothèse selon laquelle la structure IV aurait été une aire de cuisson de la céramique.

Le cas de la str. V est différent : le soubassement de pierres et les traces d'un sol stuqué d'un bâtiment très détruit ont été retrouvés sous le monticule de pierres. La céramique était ici aussi très abondante dans la couche supérieure (2338 tessons). Sous le sol, deux sépultures ont été découvertes. Dans l'une, un plat pizarra pre-Cehpech rituellement tué par une perforation en son centre recouvrait la tête du mort qui avait été enterré avec des ornements d'oreilles en céramique (en forme de fleur) recouverts de nacre.

De nombreuses questions restent donc en suspens. Nous tenons à rappeler que les résultats exposés ici sont préliminaires mais ils illustrent déjà la richesse d'informations que peuvent offrir des fouilles intensives. Ils montrent la possibilité d'approcher la vie domestique passée, les activités purement résidentielles, rituelles et peut-être artisanales. Les bâtiments entourant l'aire de captage sont probablement contemporains mais un meilleur contrôle chronologique permettrait sans doute de mieux comprendre les deux autres espaces. Ici, nous devons souligner l'importance des travaux engagés par ailleurs par Carmen Varela et Sylviane Boucher ${ }^{11}$. Ces travaux remettent en question l'unité de la céramique pizarra et posent pour principe la nécessité d'approfondir les études sur l'horizon Cehpech.

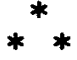

Les résultats des travaux archéologiques et de restauration à Kabah contredisent en parie l'hypothèse qui propose une occupation brève et tardive de la région 
Puuc. Si cela avait été le cas, les sites seraient formés par une seule composante temporelle couvrant une période de 200 ans. La présence de style Puuc ancien dans la majorité des sites des districts de Santa Elena et de Bolonchén va à l'encontre de cette reconstitution. Comme nous l'indique le Groupe Est, l'histoire puuc s'étend sur une séquence temporelle, marquée par l'architecture, qui couvrirait une période relativement longue et continue. Les études céramiques anciennes qui ont défini l'horizon Cehpech et lui ont assigné une durée de 250 ans, ont alimenté la vision d'un développement Puuc subit. Les recherches récentes menées par Carmen Varela et Sylviane Boucher ont montré que les techniques de fabrication de la céramique pizarra - groupe traditionnellement associé à l'horizon Cehpech - seraient déjà présentes durant le Classique Ancien. La céramique pizarra dans son ensemble ne pourrait plus être considérée comme un marqueur d'horizon. Il est à présent nécessaire d'approfondir son analyse *

\section{NOTES}

1. Le Projet Kabah, en cours actuellement, est dirigé par Ramón Carrasco V. (CRY … INAH) qui a également supervisé les travaux du Groupe Est. Durant la saison 1991 Josep Ligorred (ENAH) a commencé la restauration et l'exploration du Codz Pop et Eduardo Pérez de Heredia P. (UADY) celles de Manos Rojas. Antonio Centeno (UADY) a entrepris la reconnaissance générale du site et Fabienne de Pierrebourg (CRAP- Paris I) était chargée des fouilles de la plate-forme du Cabrio.

L'analyse céramique a été réalisée avec la collaboration de Sylviane Boucher (CRY-INAH). La présente traduction a été effectuée par Fabienne de Pierrebourg.

2. La fig. 1 a été tirée de N. Hammond (1982) Ancient Maya Civilization, Cambridge University Press, Cambridge. Le plan du centre de Kabah d'après E. M. Shook a été tiré de P. Gendrop (1977) Quince Ciudades mayas, Colección de Arte $n^{\circ}$ 31, UNAM, México D.F.

3. Pollock H. E. D., 1980. - The Puuc: an architectural survey of the Hill Country of Yucatan and Northern Campeche, Mexico. Peabody Museum of Archaeology and Ethnology, Havard University, Cambridge, Mass.

4. ANdrews G. F., 1986. - Los estilos arquitectónicos del Puuc, una nueva apreciation. Colección Cientifica $n^{\circ} 150$, UNAM, México D.F. D.F.

5. Gendrop P., 1983. - Los estilos Río Bec, Chenes y Puuc en la arquitectura maya, UNAM, México

6. Exposées au Musée d'Anthropologie de Mexico D.F et au Musée du Palacio Canton de Mérida (Yuc.)

7. Stephens J.-L., 1986. - Incidents of travel in Yucatcin, vol. II, Editorial Dante, Mérida, Yucatán.

8. Proskouriakoff T., 1950. - A study of maya classic sculpture, Pub. 593, Carnegie Institution of Washington, Washington D.C.

9. SCHELE L. et D. FReidel, 1990. - A Forest of Kings. The untold story of the ancient Maya. William Morrow and $\mathrm{C}^{\circ}$ Inc., N.Y.

10. Ramón Carrasco, 1991. -- "Formación sociopolitica en el Puuc : El sacbé Uxmal- NohpatKabah ", Communication orale, Tercera Mesa Redonda de la Socieda Española de Estudios Mayas, Girona.

11. Les travaux en cours sur la céramique pizarra et ses antécédents ont été l'objet de plusieurs communications orales de la part de Sylviane Boucher et Carmen Varrela; certaines de ces communications devraient être publiées prochainement.

* Manuscrit reçu en mars 1992, accepté pour publication en mai 1992. 